
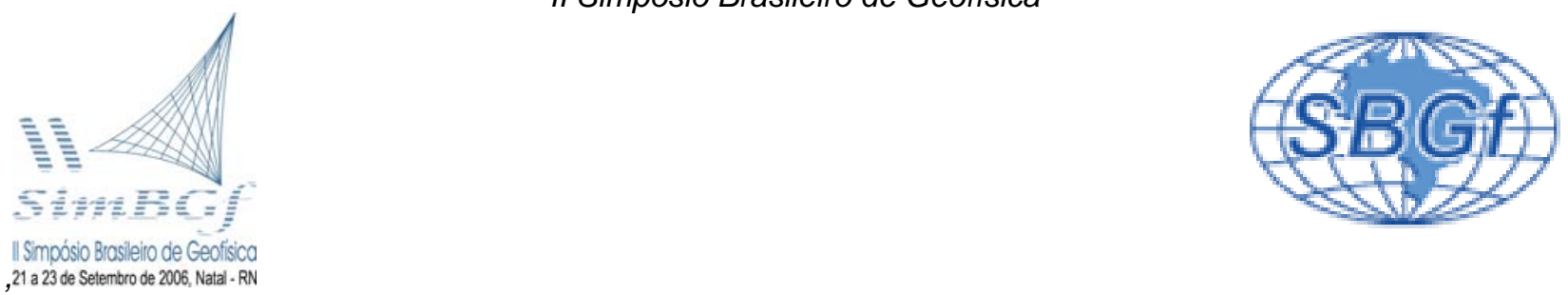

\title{
Migração FD e FFD usando aproximação de Padé complexa
}

Daniela Amazonas, UFPA

Jessé Costa, UFPA

Copyright 2006, SBGf - Sociedade Brasileira de Geofísica

Este texto foi preparado para a apresentação no II Simpósio de Geofísica da Sociedade Brasileira de Geofísica, Natal, 21-23 de setembro de 2006. Seu conteúdo foi revisado pela Comissão Tecno-científica do II SR-SBGf mas não necessariamente representa a opinião da SBGf ou de seus associados. E proibida a reprodução total parcial deste material para propósitos comerciais sem prévia autorização da SBGf.

\section{Resumo}

Apresentamos dois algoritmos estáveis para a migração por diferenças finitas e migração híbrida por Fourier finite difference. Os algoritmos utilizam expansão de Padé complexa para aproximar o operador de raiz quadrada associado à equação da onda unidirecional. $\mathrm{O}$ algoritmo proposto de migração apresenta resposta ao impulso semelhante à migração PSPI, como indicam simulações da resposta ao impulso e de migração pósempilhamento usando o modelo Marmousi. O algoritmo também foi utilizado para migração pré-empilhamento apresentando resultados satisfatórios e a um custo computacional muito menor que o método Fourier finite difference plus interpolation.

\section{Introdução}

Algoritmos de migração baseados na equação de onda apresentam melhor desempenho na presença de forte variação lateral de velocidade. As migrações por diferenças finitas, phase shift, split-step, phase screen e a migração por Fourier finite difference são abordagens para migração usando a equação da onda.

A migração phase-shift é um método eficiente e preciso de propagação com grandes ângulos, se a velocidade variar somente com a profundidade. Já a migração phase-shift plus interpolation (PSPI) é mais tolerante a variações de velocidade. Por outro lado, a migração por diferenças finitas permite modelos com variações laterais de velocidade, mas apresenta limitações para ângulos grandes de incidência e ondas evanescentes. Ristow e Rühl (1994), propuseram um algoritmo híbrido de diferenças finitas, phase-shift e splitstep denominado Fourier finite difference (FFD), que apresenta vantagens em relação a cada um destes métodos aplicados isoladamente, particularmente em modelos com fortes variações laterais de velocidade.

Infelizmente, quando o método FFD é aplicado na presença de descontinuidades acentuadas no modelo de velocidade, apresenta instabilidade numérica. Para corrigir esta limitação Biondi (2002) propôs modificações ao algoritmo FFD que o torna incondicionalmente estável, o qual denominou Fourier finite difference plus interpolation (FFDPI). Este algoritmo requer interpolação do campo de velocidades o que aumenta o seu custo computacional em relação ao algoritmo FFD.

Zhang et al (2003) aplicou uma técnica com aproximações de Padé complexa para o método de migração 3D por diferenças finitas. Todavia, esse método não se mostrou eficiente para propagação de grandes ângulos. Ainda utilizando a aproximação de Padé complexa Zhang et al (2004) apresentou o método de migração split-step complex Padé Fourier 3D que está baseado no operador exponencial exato multiplicando e dividindo pelo exponencial aproximado que é usado na solução de Fourier.

Utilizando a expansão de Padé complexa (Milinazzo et al, 1997) para aproximar o operador de raiz quadrada da equação da onda unidirecional, apresentamos um algoritmo de migração $2 \mathrm{D}$ por diferenças finitas e um outro de migração FFD também 2D que apresentam maior estabilidade que a implementação FFD original e desempenho equivalente ao algoritmo FFDPI com custo computacional menor.

\section{Metodologia}

Os algoritmos a seguir utilizam aproximações racionais para a equação da onda unidirecional:

$$
\frac{\partial P(\bar{x}, \omega)}{\partial x_{3}}=\frac{(-i \omega)}{c(\bar{x})} \sqrt{1+\frac{c^{2}(\bar{x})}{\omega^{2}} \frac{\partial^{2}}{\partial x_{1}^{2}}} P(\bar{x}, \omega),
$$

em que $P(x, \omega)$ é o campo de pressão, $c(\bar{x})$ é a velocidade e $i=\sqrt{-1}$. Para aproximar a raiz quadrada da Eq. (1) nós utilizamos aproximações da forma:

$$
\sqrt{1+z}=e^{i \alpha / 2} \sqrt{1+\left[(1+z) e^{-i \alpha}-1\right]} \approx R_{N}(z)=C_{0}+\sum_{n=1}^{N} \frac{A_{n} z}{1+B_{n} z},
$$

em que

$$
\begin{gathered}
C_{0}=e^{i \alpha / 2}\left(1+\sum_{n=1}^{N} \frac{a_{n}\left[e^{-i \alpha}-1\right]}{1+b_{n}\left(e^{-i \alpha}-1\right)}\right), \quad z=\frac{c^{2}(\bar{x})}{\omega^{2}} \frac{\partial^{2}}{\partial x_{1}^{2}}, \\
A_{n}=\frac{e^{-i \alpha / 2} a_{n}}{\left[1+b_{n}\left(e^{-i \alpha}-1\right)\right]^{2}} \quad \text { e } \quad B_{n}=\frac{b_{n} e^{-i \alpha}}{1+b_{n}\left(e^{-i \alpha}-1\right)} .
\end{gathered}
$$

$A_{n}$ e $B_{n}$ são coeficientes de Padé complexos propostos por Milinazzo et al (1997), $a_{n}$ e $b_{n}$ são os coeficientes da expansão de Padé real (Bamberger et al, 1988) e são dados por 


$$
a_{n}=\frac{2}{2 N+1} \operatorname{sen}^{2} \frac{n \pi}{2 N+1} \quad \text { e } \quad b_{n}=\cos ^{2} \frac{n \pi}{2 N+1}
$$



Figura 1: Esquema do plano z e da linha de corte

No plano complexo, a expansão de Padé com coeficientes reais corresponde às aproximações da raiz quadrada com linhas de corte ao longo do eixo real negativo. A introdução da fase $\alpha$ na Eq. (2) rotaciona a linha de corte e desloca o ponto de ramificação da raiz da origem para o ponto $(-1,0)$ conforme mostra a Fig. 1 . Esta modificação resulta em uma melhor representação da parte evanescente do espectro, desta forma melhorando a estabilidade da aproximação.

Substituindo (2) em (1) temos:

$$
\frac{\partial P(\bar{x}, \omega)}{\partial x_{3}}=\frac{-i \omega}{c(\bar{x})}\left[C_{0}+\sum_{n=1}^{N} \frac{A_{n} \frac{c^{2}(\bar{x})}{\omega^{2}} \frac{\partial^{2}}{\partial x_{1}^{2}}}{1+B_{n} \frac{c^{2}(\bar{x})}{\omega^{2}} \frac{\partial^{2}}{\partial x_{1}^{2}}}\right] p(\bar{x}, \omega) .
$$

A implementação da Eq. (3) resulta no algoritmo de migração por diferenças finitas com aproximação de Padé complexa.

Seguindo metodologia semelhante a utilizada por Ristow e Rühl (1994), deduzimos a seguinte aproximação para a migração FFD utilizando expansão de Padé complexa:

$\frac{-i \omega}{c(\bar{x})} \sqrt{1+\frac{c^{2}(\bar{x})}{\omega^{2}} \frac{\partial^{2}}{\partial x_{1}^{2}}} \approx \frac{-i \omega}{c_{r}} \sqrt{1+\frac{c_{r}^{2}}{\omega^{2}} \frac{\partial^{2}}{\partial x_{1}^{2}}} P(\bar{x}, \omega)+$

$(i \omega)\left(\frac{1}{c(\bar{x})}-\frac{1}{c_{r}}\right) C_{0} P(\bar{x}, \omega)+$

$(-i \omega)\left[\sum_{n=1}^{N} \frac{A_{n}\left(\frac{c-c_{r}}{\omega^{2}}\right) \frac{\partial^{2}}{\partial x_{1}^{2}}}{1+2 B_{n}\left(\frac{c^{2}+c_{r}^{2}}{\omega^{2}}\right) \frac{\partial^{2}}{\partial x_{1}^{2}}}\right] P(\bar{x}, \omega)$

em que a velocidade de referência $C_{r}$ é a velocidade mínima em cada nível do processo de continuação do campo de ondas para baixo. $\mathrm{Na}$ próxima secção apresentamos os resultados dos dois algoritmos de migração, baseados nas Eqs. (3) e (4) e comparamos com os resultados de outros algoritmos de migração em profundidade utilizando continuação para baixo do campo de ondas.

\section{Resposta ao impulso no modelo Marmousi}

Para avaliar o desempenho dos algoritmos utilizando aproximação de Padé complexa comparamos a resposta ao impulso dos mesmos com os seguintes algoritmos de migração por continuação para baixo do campo de ondas: FD, FFD, PSPI e FFDPI. Exceto o algoritmo FFDPI, utilizamos as implementações do pacote SU (Cohen e Stockwell, 2005). A resposta ao impulso foi avaliada no modelo Marmousi, para um pulso Ricker com freqüência pico de $25 \mathrm{~Hz}$, para um traço na posição central do modelo, no instante $0,8 \mathrm{~s}$. Para realçar os eventos, aplicamos o mesmo ganho a todos os resultados.

A Fig. 2 mostra a resposta ao impulso da migração por diferenças finitas para grande abertura. A Fig. 3 mostra a resposta ao impulso da migração FD utilizando a Eq. (3), a rotação da linha de corte foi de 90 graus. A redução da dispersão numérica no resultado com a aproximação de Padé complexa é evidente e decorre do tratamento mais adequado para as ondas evanescentes, como indica Milinazzo et al (1997) e Yevick et al (2000). A resposta ao impulso do algoritmo proposto na Eq. (4) está apresentada na Fig. 5. Comparando com a resposta ao impulso do algoritmo FFD original, Fig. 4, o algoritmo proposto apresenta maior qualidade na imagem e menor dispersão numérica.

Para melhor avaliar a resposta do novo algoritmo comparamos seu resultado com a resposta ao impulso da migração PSPI, Fig. 6, e a resposta ao impulso usando o algoritmo FFDPI proposto por Biondi (2002), Fig. 7. Observa-se maior semelhança entre a resposta ao impulso destes três algoritmos do que com a resposta ao impulso do algoritmo FFD original. Entretanto, o algoritmo proposto não requer nenhuma interpolação de migrações feitas com diferentes velocidades de referência. Esta característica é responsável pelo seu menor custo computacional em relação ao algoritmo FFDPI.

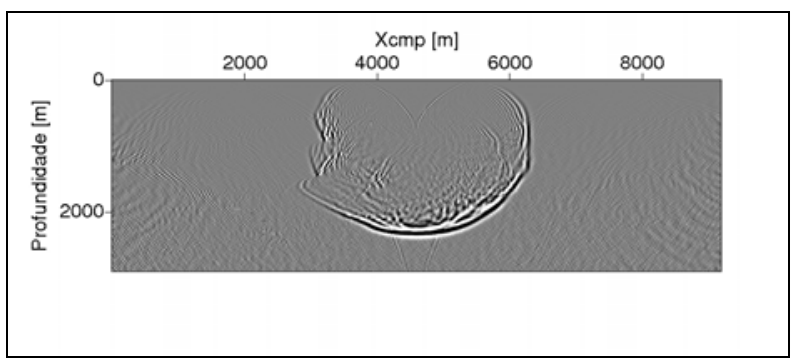

Figura 2: Resposta ao impulso utilizando o método FD convencional

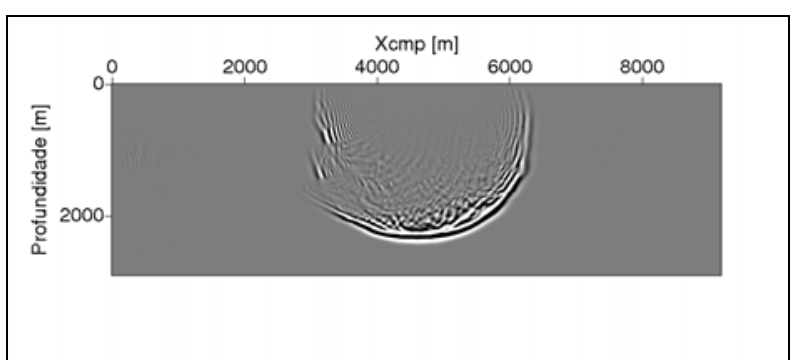

Figura 3: Resposta ao impulso utilizando o método FD com aproximação de Padé complexa 




Figura 4: Resposta ao impulso utilizando o método FFD original

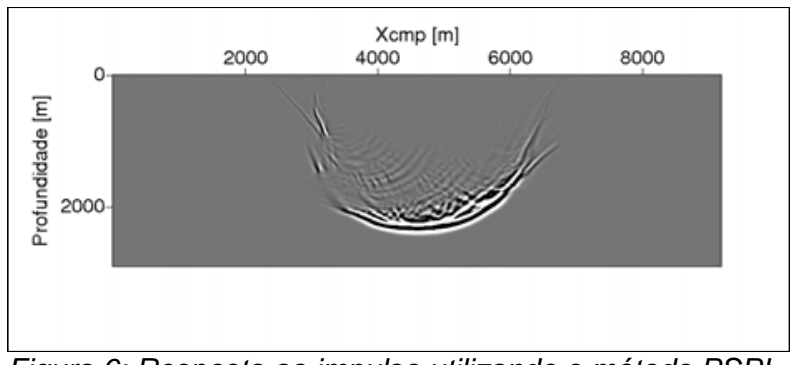

Figura 6: Resposta ao impulso utilizando o método PSPI

\section{Migração Pré-empilhamento dos dados Marmousi}

Como segundo experimento numérico, efetuamos a migração pré-empilhamento no modelo Marmousi no domínio de tiro comum. O resultado da migração com as implementações descritas acima foram comparados aos métodos FFDPI, PSPI, FFD e FD. Novamente, com exceção do algoritmo FFDPI, foi utilizada a implementação do pacote Seismic Unix. Utilizamos a prescrição de Bagaini et al (1995) na escolha de velocidades para o termo de deslocamento de fase em nossa implementação do algoritmo FFDPI.

A condição de imagem em todos os algoritmos foi a correlação entre o campo incidente e o campo retropropagado. Para todos os exemplos a seguir o campo incidente foi calculado usando um pulso Ricker com freqüência pico de $25 \mathrm{~Hz}$. A banda de freqüência utilizada foi de 0 a $60 \mathrm{~Hz}$. Nenhum pré-processamento foi aplicado aos dados de entrada. Utilizamos oito parcelas da expansão nas equações (3) e (4).

A Fig. 8 mostra o resultado da migração utilizando o método FD original enquanto a Fig. 9 mostra os dados migrados pelo método FD com aproximação de Padé complexa. É evidente a melhoria na definição dos refletores. A diferença na freqüência do sinal deve-se ao fato de nas implementações do SU termos usado uma janela Hamming com freqüências de $5 \mathrm{~Hz}, 10 \mathrm{~Hz}, 40 \mathrm{~Hz}$ e $50 \mathrm{~Hz}$.

A Fig. 10 mostra o método FFD original que comparado ao nosso algoritmo FFD, Fig. 11, apresenta menor qualidade de definição nas falhas estruturais do modelo. Comparando ainda a Fig. $11 \mathrm{com}$ a Fig. 12, método PSPI, é perceptível a dificuldade do método PSPI em imagear estruturas até aproximadamente 1500m em profundidade enquanto nosso algoritmo FFD mostra essas estruturas com uma boa definição.

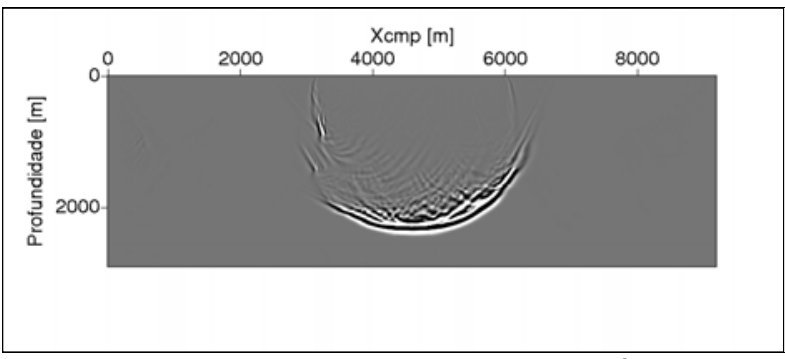

Figura 5: Resposta ao impulso utilizando o método FFD com aproximação de Padé complexa

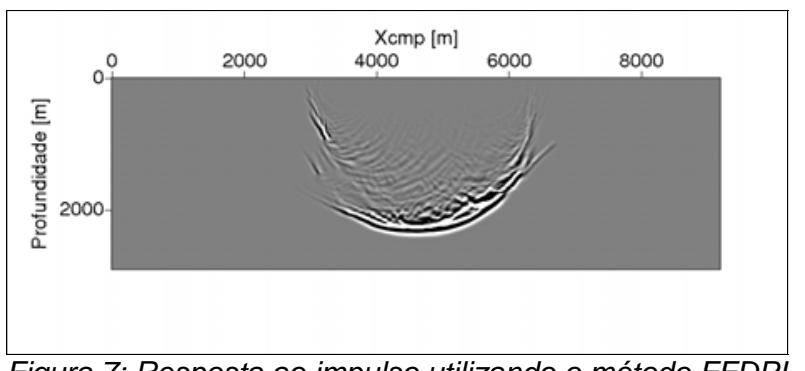

Figura 7: Resposta ao impulso utilizando o método FFDPI

Ao observar a Fig. 13 que mostra a aplicação do método FFDPI e a Fig. 11, é notória a semelhança entre elas, com exceção das áreas $\mathrm{A}, \mathrm{B}$ e $\mathrm{C}$, o que a ressalta a qualidade do método FFDPI.

A Fig.14 mostra o modelo de velocidade do dado Marmousi. Comparando os resultados de todos os métodos de migração que utilizamos neste trabalho com a Fig. 14 notamos que nosso algoritmo consegue imagear o reservatório (área $\mathrm{D}$ ) com resolução semelhante ao método FFDPI.

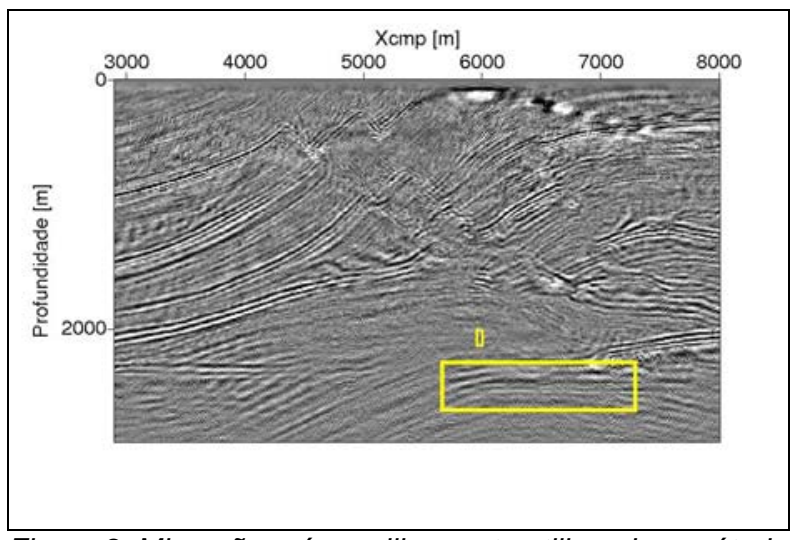

Figura 8: Migração pré-empilhamento utilizando o método FD original. 


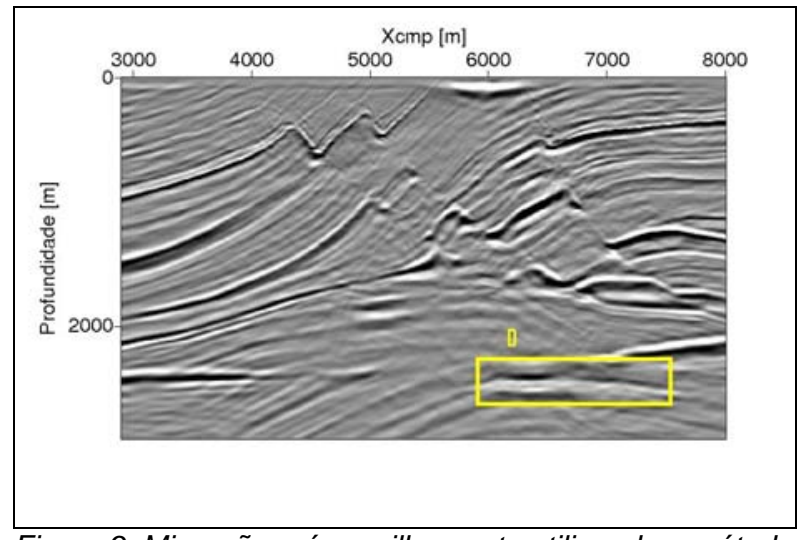

Figura 9: Migração pré-empilhamento utilizando o método FD com aproximação de Padé complexa.

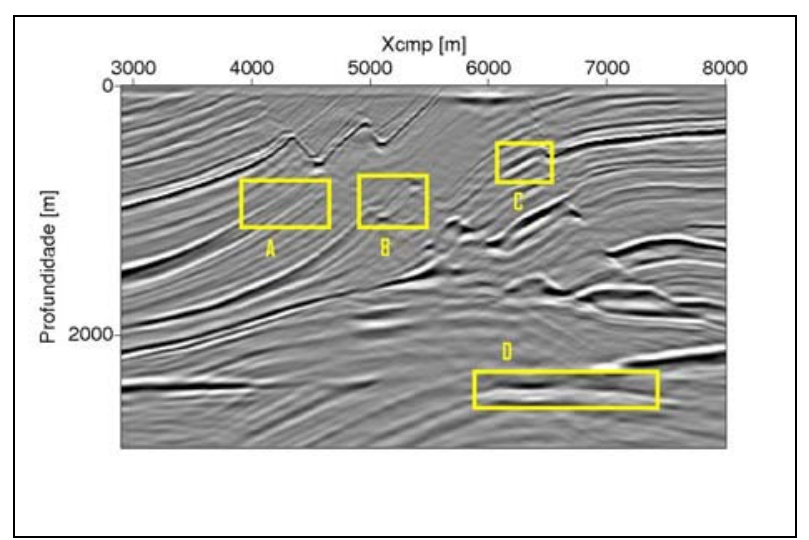

Figura 11: Migração pré-empilhamento utilizando o método FFD com aproximação de Padé complexa.

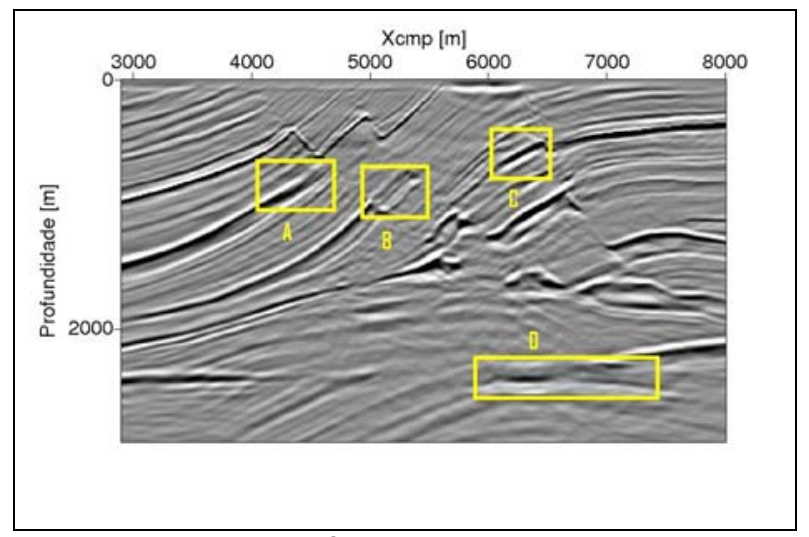

Figura 13: Migração pré-empilhamento utilizando o método FFDPI.

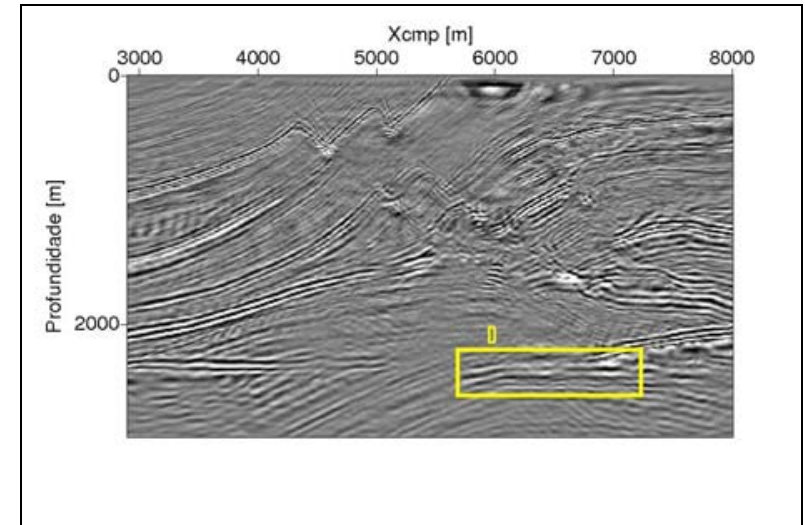

Figura 10: Migração pré-empilhamento utilizando o método FFD original.

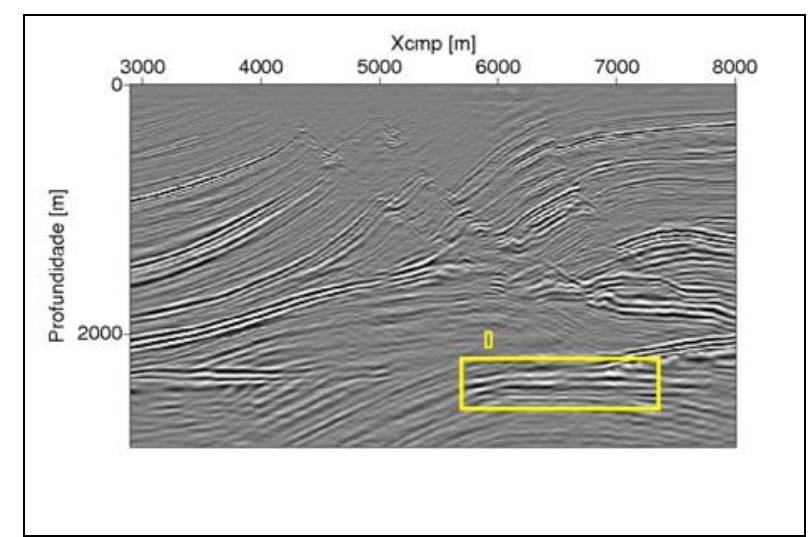

Figura 12: Migração pré-empilhamento utilizando o método PSPI.



Figura 14: Modelo de velocidade Marmousi 


\section{Discussão e Conclusões}

Para modelar fortes variações laterais de velocidade, a combinação da precisão dos métodos de Fourier para migração de eventos com forte inclinação com a flexibilidade de diferenças finitas implícita produz métodos de continuação para baixo precisos e eficientes.

A correção FFD é a mais eficaz que a migração por diferenças finitas, pois inclui termos de deslocamento de fase, split-step além de termos de difração calculados por diferenças finitas.

A avaliação da resposta impulsiva do algoritmo proposto, no modelo de velocidade Marmousi, foi comparada a resposta impulsiva da migração por deslocamento de fase, da migração por diferenças finitas, da migração FFD e FFDPI . Os resultados mostram que a resposta impulsiva da migração FFD usando aproximação de Padé complexa se aproxima da migração por deslocamento de fase e da migração FFDPI, o que sugere que o operador pode migrar eventos com forte mergulho.

Os resultados da migração pré-empilhamento usando FFD com aproximação de Padé complexa indicam que esta abordagem melhora a estabilidade do algoritmo original e apresenta resultados semelhantes ao método FFDPI a um custo computacional muito inferior.

\section{Agradecimentos}

Agradecemos o financiamento oferecido através do projeto patrocinado por FINEP/CNPq/PETROBRAS e ao CNPq pela bolsa de mestrado.

\section{Referências}

BAGAINI, C., BONOMI, E., PIERONI, E. 1995. Data parallel implementation of 3-D PSPI. $65^{\text {th }}$ Ann. Internat. Mtg. Soc. Expl. Geophys. , Expanded Abstracts, 188-191.

BAMBERGER, A., et al, 1988. Higher order paraxial wave equation approximations in heterogeneous media. $\mathrm{J}$. Appl. Math.,48: 129-154.

BIONDI, B., 2002. Stable wide-angle Fourier finitedifference downward extrapolation of 3-D wavefields. Geophysics, 67: 872-882

COHEN, J. K. \& STOCKWELL, Jr. J. W. 2005. CWP/SU: Seismic Unx Release n. 39 a free package for seismic research e processing, Center for Wave Phenomena, Colorado School of Mines.

MILINAZZO, F. A., ZALA, C. A. \& BROOKE, G. H. 1997. Rational square-root approximations for parabolic equation algorithms. J. Acoust. Soc. Am., vol. 101, n. 2: 760-766.

RISTOW, D. \& RÜHL, T., 1994. Fourier finite-difference migration. Geophysics, 59: 1882-1893.
YEVICK, D. \& THOMSON, D. J. 2000. Complex Padé approximants for wide-angle acoustic propagators. J. Acoust. Soc. Am., vol. 108: 2784-2790.

ZHANG, L., RECTOR, J.W.,HOVERSTEN, G. M., \& FOMEL, S. 2004. Split-Step complex Padé-Fourier Depth migration. SEG Int'l Exposition and 74th Annual Meeting. Denver, Colorado. 